\section{(2) \\ OPEN ACCESS}

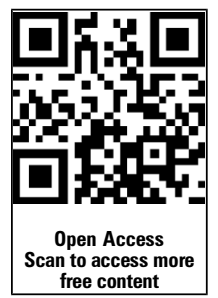

${ }^{1}$ Division of Population, Health Sciences and Education, St George's University of London, Cranmer Terrace, London, UK

${ }^{2}$ Department of Palliative Medicine, Royal Derby Hospital, Derby, UK

${ }^{3}$ Department of primary care and community health, School of Nursing, Midwifery and Social Work, University Place, The University of Manchester, Manchester, UK ${ }^{4}$ Department of Palliative Medicine, Palliative Care Team, Surrey and Sussex Healthcare NHS Trust, East Surrey Hospital, Redhill, Surrey, UK

${ }^{5}$ Department of Public Health and Primary Care, Primary Care Unit, Institute of Public Health, Cambridge, UK

\section{Correspondence to}

Dr Patrick C Stone, Division of Population, Health Sciences and Education, St George's University of London, Cranmer Terrace, London SW17 ORE, UK; pstone@sgul.ac.uk

Received 25 October 2012 Accepted 6 December 2012 Published Online First

7 January 2013

\section{SLinked}

- http://dx.doi.org/10.1136/ bmjspcare-2013-000461

To cite: Stone $P C$, Gwilliam B, Keeley V, et al. BMJ Supportive \& Palliative Care 2013;3:318-323.

\title{
Factors affecting recruitment to an observational multicentre palliative
} care study

\author{
Patrick C Stone, ${ }^{1}$ Bridget Gwilliam, ${ }^{1}$ Vaughan Keeley, ${ }^{2}$ Chris Todd, ${ }^{3}$ \\ Laura C Kelly, ${ }^{4}$ Stephen Barclay ${ }^{5}$
}

\begin{abstract}
Objectives To identify those factors which adversely affected recruitment to a large multicentre palliative care study.
\end{abstract}

Methods Patient accrual to a multicentre, observational, palliative care study was monitored at three critical junctures in the research process. (1) Eligibility-did the patient fulfil the study entry criteria? (2) Accessibilitywas it possible to access the patient to be able to inform them about the study? (3) Consent-did the patient agree to participate in the study? The reasons why patients were ineligible, inaccessible or refused consent were recorded.

Results 12412 consecutive referrals to participating clinical services were screened for study inclusion of whom 5394 (43\%) were deemed to be ineligible. Of the remaining patients $4617 / 7018$ (66\%) were inaccessible to the research team. The most common reasons being precipitous death, 'gatekeeping' by clinical staff or rapid discharge. Of the 2410 patients who were visited by the research team and asked to participate in the study 1378 (57\%) declined. Overall $8.2 \%(1018 / 12412)$ of patients screened participated in the study. There were significant differences in recruitment patterns between hospice inpatient units, hospital support and community palliative care teams.

Conclusions Systematic monitoring and analysis of patient flows through the clinical trial accrual process provided valuable insights about the reasons for failure to recruit patients to a clinical trial and may help to improve recruitment in future studies.

\section{BACKGROUND}

It is often stated that recruitment to palliative care studies poses particular challenges. ${ }^{12}$ These include the nature of the patient population, the high prevalence of cognitive problems, the unstable nature of the disease process and the lack of research infrastructure and experience in palliative care teams. 'Gatekeeping' (ie, preventing access to potential research subjects) by clinical staff has also been cited as a problem in palliative care research studies. ${ }^{3} 4$ As a result, and despite there being evidence that palliative care patients and their carers are frequently in favour of participating in research, ${ }^{5}$ many studies fail to obtain their objective due to difficulties with recruiting the required sample size. ${ }^{7}$

A number of research groups have reported on the difficulties that they have encountered in recruiting participants to palliative care studies. ${ }^{2-10}$ One report ${ }^{8}$ described the barriers to recruitment to clinical trials at the palliative care unit of the Royal Marsden Hospital, UK. Over a 4-year period 1206 patients were considered for inclusion into 23 separate clinical trials. It was reported that the most common reasons given for refusal to participate in research projects were a wish to defer to a later date, deterioration in clinical condition or excessive travelling distance to the hospital concerned. Other reports of researchers' experience have involved far fewer patients. A systematic review ${ }^{6}$ identified 11 papers investigating the attitudes of patients with advanced cancer to participation in research studies. The median sample size for these studies was 33 subjects and the largest study included 214 participants.

The Prognosis in Palliative care Study (PiPS) was a large multicentre observational study to develop a novel prognostic indicator for use with patients with advanced cancer. ${ }^{9}$ As we were aware that many previous studies in palliative care populations had failed to recruit the intended sample size, we prospectively 
collected information on difficulties that we anticipated encountering in patient accrual. In this paper we describe the difficulties that were encountered in identifying, approaching and consenting potentially eligible patients.

\section{METHODS}

Recruitment to the PiPS study took place in 18 palliative care services across England and included hospices, hospital support and community palliative care teams.

Patients were eligible to participate in the PiPS study if they had locally advanced or metastatic cancer and were no longer undergoing disease-modifying treatment. Both competent and non-competent patients were eligible. Competent patients were required to complete some simple questionnaires and provide a blood sample for routine laboratory analysis. Non-competent patients were not required to provide a blood sample or complete any questionnaires, but relevant information was extracted from their medical records and an observer-rated symptom checklist was completed by clinical staff. Competent patients gave written informed consent. In the case of noncompetent patients the assent of a carer or relative was required. In order to protect vulnerable patients from any perceived pressure to participate in the research process, study staff was required to gain permission from the clinical team prior to approaching individuals. Further details of inclusion and exclusion criteria have been published elsewhere. ${ }^{9}$

To monitor recruitment rates and identify barriers to accrual, an electronic screening log was developed using EXCEL 2003. Every patient referred to participating palliative care services was systematically considered for inclusion and was entered onto the screening log. The subsequent 'journey' of potentially eligible participants through the recruitment process was monitored and when possible the reasons for failure to recruit were recorded at each of three key potential 'bottlenecks'.

1. Eligibility-The main reasons for ineligibility were coded as; non-cancer, still receiving cancer treatment, no evidence of advanced disease, under 18 years old, unable to understand sufficient English, other reasons. ${ }^{9}$

2. Accessibility-The main reasons for failure of the research staff to access potentially eligible patients were coded as; researcher being unavailable (eg, annual leave), patient died or was discharged before being approached by research staff, patient not available when research staff visited (eg, undergoing investigations), relatives or carers of non-competent patient not available, 'gatekeeping' by clinical staff, patient refused permission for research staff to discuss study, other reasons.

3. Consent-Competent patients or the carers/relatives of non-competent patients were under no obligation to provide any reasons for non-participation in the research. However, many were happy to do so. Their responses were coded as; not wishing to contemplate prognosis, not wishing to have a blood test, too many competing priorities to think about research, patient deteriorated too quickly between initial discussion and consent being obtained, patient too fatigued, other reasons.

\section{Statistical methods}

Data were summarised using descriptive statistics (percentages, proportions, means and standard deviations). The $\chi^{2}$ test ${ }^{10}$ was used to compare the proportions of potential participants who were found to be ineligible, inaccessible or refused to participate according to whether the patients were screened in hospices, hospitals or community teams. Unpaired $t$ tests were used to compare means.

\section{RESULTS}

Between March 2006 and August 2009, 12412 patients were screened for eligibility for PiPS (hospices $\mathrm{n}=4593$; hospitals $\mathrm{n}=4696$; community teams $\mathrm{n}=3123$ ). Of these patients only 1018/12 412 (8\%) subjects were actually recruited to the study. Figure 1 shows the flow of patients through the recruitment process and the attrition at each of the three key bottlenecks. Of the 12412 patients screened, 7018 (57\%) were eligible for inclusion, 2401/7018 (34\%) of those patients were accessed by the research team and of those 1023/2401 (43\%) were subsequently recruited to the study.

\section{Eligible versus ineligible patients}

There were significant differences in the proportion of eligible patients in each of the recruitment areas

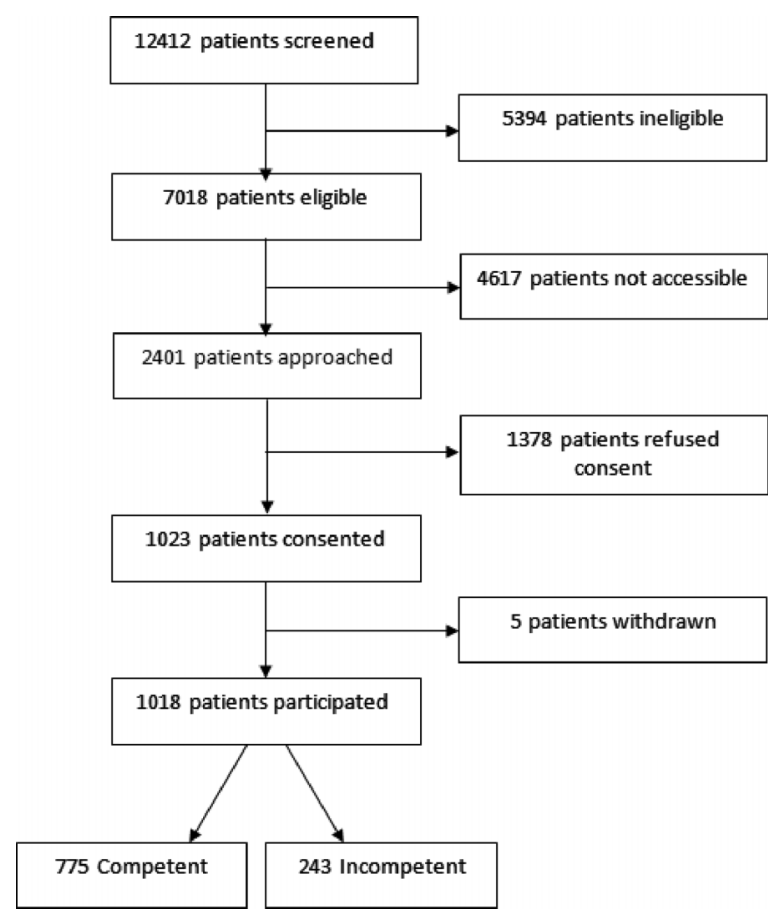

Figure 1 CONSORT diagram to show recruitment to the Prognosis in Palliative care Study (PiPS) study. 
Table 1 Reasons for ineligibility by site of screening

\begin{tabular}{|c|c|c|c|c|}
\hline Reason for ineligibility & Community, n (\%) & Hospice, n (\%) & Hospital, n (\%) & Total n (\%) \\
\hline Still receiving treatment & $1055(76 \%)$ & $384(33 \%)$ & $1820(64 \%)$ & $3259(60 \%)$ \\
\hline Non-cancer diagnosis & $185(13 \%)$ & $460(39 \%)$ & $821(29 \%)$ & $1466(27 \%)$ \\
\hline Previously screened & $75(5 \%)$ & $249(21 \%)$ & $67(2 \%)$ & $391(7 \%)$ \\
\hline Poor English & $34(2 \%)$ & $55(5 \%)$ & $58(2 \%)$ & $147(3 \%)$ \\
\hline$<18$ years old & $1(<1 \%)$ & $0(0 \%)$ & $8(<1 \%)$ & $9(<1 \%)$ \\
\hline No evidence of advanced cancer & $25(2 \%)$ & $17(1 \%)$ & $67(2 \%)$ & $109(2 \%)$ \\
\hline Other & $12(<1 \%)$ & $0(0 \%)$ & $1(<1 \%)$ & $13(<1 \%)$ \\
\hline Total & $1387(100 \%)$ & $1165(100 \%)$ & $2842(100 \%)$ & $5394(100 \%)$ \\
\hline
\end{tabular}

(74.6\% hospice patients, $39.5 \%$ hospital, $55.6 \%$ community; $\mathrm{p}<0.000001)$. The mean age of eligible patients was 72.2 years (SD 12.5) and 47.7\% (3348/ 7018) were female. Table 1 shows the reasons why patients were ineligible to be considered for the PiPS study. The most common reason for being ineligible was that the patient was still receiving (or being considered for) cancer treatment (3259/5394; 60\%). There were significant differences in the proportion of ineligible patients who were identified as still on active treatment in each of the research settings (33\% in hospice, $64 \%$ hospital and $76 \%$ community; $\mathrm{p}<0.000001)$. The next most common reason for being ineligible was being a patient with a non-cancer diagnosis $(1466 / 5394 ; 27 \%)$. This was most likely to occur in hospice patients and least likely in the community (39\% hospice, 29\% hospital, 13\% community; $\mathrm{p}<0.000001)$.

\section{Accessible versus inaccessible patients}

It was possible for the research team to access 2401/ 7018 (34\%) of the eligible patients. Patients were significantly $(\mathrm{p}<0.000001)$ easier to access in hospices $(47 \%)$ than in hospitals $(22 \%)$ and the community $(22 \%)$. The mean age of accessible patients was 72.2 years (SD 11.8) and $47.6 \%$ were female (1144/ 2401). This was not significantly different from the age or gender distribution of inaccessible patients (age 72.2 years, $47.7 \%$ female).

Table 2 shows the reasons why patients could not be accessed by the research team. The most common reason why patients could not be accessed was that they died before there was an opportunity to inform them or their relatives about the study. Early death as a reason for not being recruited to the study was most common among community patients and least common in hospital patients (hospice 25\%, hospital $18 \%$, community $30 \% ; \mathrm{p}<0.000001)$. The second most common reason for patients not being accessed by research staff was 'gatekeeping' by clinical staff. Gatekeeping was significantly $(p=0.0025)$ less common in hospice patients $(22 \%)$ but was not significantly different between hospital (25\%) and community (26\%) patients. Gatekeeping could be due to clinical staff judging that the research would cause the patient $(62 \%$ of occasions when gatekeeping occurred) or their relative $(26 \%)$ distress or if it was judged that the member of staff would find it too challenging to raise the research project with the patient/family (12\%). A number of eligible patients $(503 ; 11 \%)$ were rendered inaccessible because they were non-competent and either had no carers to provide assent or their carers visited at times (eg, evenings or weekends) when a researcher was not available to discuss the study with them. Researchers were often covering several sites and could not always guarantee being available to discuss the study with a patient within 1 week of referral as required by the protocol. This occurred in 344 (7\%) cases.

\section{Consenters versus non-consenters}

Of the 2401 patients who were approached by the research staff 1023 (43\%) consented to participate in the study (representing $15 \%$ of all eligible patients).

Table 2 Reasons for inaccessibility by site of screening

\begin{tabular}{|c|c|c|c|c|}
\hline Reason for inaccessibility & Community, n (\%) & Hospice, n (\%) & Hospital, n (\%) & Total n (\%) \\
\hline Died before review & $409(30 \%)$ & $457(25 \%)$ & $260(18 \%)$ & $1126(24 \%)$ \\
\hline Gatekeeping & $352(26 \%)$ & $390(22 \%)$ & $359(25 \%)$ & $1101(24 \%)$ \\
\hline Discharged before review & $281(21 \%)$ & $126(7 \%)$ & $366(25 \%)$ & $773(17 \%)$ \\
\hline Patient did not wish to see researcher & $217(16 \%)$ & $212(12 \%)$ & $149(10 \%)$ & $578(13 \%)$ \\
\hline Non-competent patient and relatives unavailable & $34(2 \%)$ & $233(13 \%)$ & $236(16 \%)$ & $503(11 \%)$ \\
\hline Researcher unavailable & $7(1 \%)$ & $297(17 \%)$ & $40(3 \%)$ & $344(7 \%)$ \\
\hline Patient unavailable & $22(2 \%)$ & $63(4 \%)$ & $15(1 \%)$ & $100(2 \%)$ \\
\hline Other & $40(3 \%)$ & $22(1 \%)$ & $30(2 \%)$ & $92(2 \%)$ \\
\hline Total & $1362(100 \%)$ & $1800(100 \%)$ & $1455(100 \%)$ & $4617(100 \%)$ \\
\hline
\end{tabular}


Community patients were significantly $(\mathrm{p}=0.008)$ less likely to consent $(36 \%)$ than hospice $(43 \%)$ or hospital patients $(46 \%)$. The mean age of consenting patients was 71.7 years (SD 11.8) and this was not significantly different from the mean age of patients who refused consent (72.6; SD 11.8 years). A slightly lower proportion of consenters than non-consenters were female $(45.2 \%$ vs $49.5 \%$; $\mathrm{p}=0.036)$.

Patients were under no obligation to provide any reason for non-participation in the study and most patients did not give a specific reason. Only 86 patients reported that they did not consent because they did not want to have further venepuncture.

\section{DISCUSSION}

\section{Statement of principal findings}

We identified three main 'bottlenecks' to recruitment; eligibility, accessibility and consent. A higher than expected number of 'palliative care' patients was ineligible because they were still receiving active cancer treatment (26\%). This probably reflects the nature of palliative care practice in the UK, with many patients being referred to services while still receiving lifeprolonging treatments in order, for instance, to provide symptom control advice or advance care planning. We found that $37 \%$ of patients were 'inaccessible' to the research team. Some of these patients could probably never have been included in any study (eg, those that died or were discharged before being approached by research staff) but $9 \%$ of patients were denied the opportunity to participate in research because of 'gatekeeping' by clinical staff. Approximately half of patients or relatives who were informed about the study agreed to participation. Hospice inpatients were most likely to be eligible for the study and were most likely to be accessed by the research team. Hospital patients were most likely to consent to participate.

\section{Strengths of the study}

This is the largest report that we are aware of that has systematically presented information about the reasons why patients could or could not be recruited to a palliative care study. Data were collected in 18 different locations, comprising inpatient, hospital and community settings. By systematically recording information about all of the patients referred to the participating units during the duration of the study it was possible to quantify the extent to which gatekeeping and other factors influenced poor accrual. It was also possible to make comparisons between the effectiveness of recruitment in different settings (ie, hospice, hospital or community).

\section{Weaknesses of the study}

Due to the large numbers of patients screened and the number of centres involved, only limited data could reliably be collected. The 'coding' of reasons for failure to accrue patients to the study was the responsibility of research staff in the participating units and it was not possible to objectively validate the veracity of these reports. It is thus possible, for instance, that research staff may have under-reported or over-reported the true extent of 'gatekeeping'. Once patients or relatives were accessed by research staff and informed about the PiPS study they were under no obligation to consent to participate or to provide a reason for deciding not to do so. In any event the reasons why patients chose not to participate are likely to be complex and perhaps could have been better addressed using a qualitative research methodology. However, this was beyond the resources of the current project.

Another weakness of the study was the lack of detail about the reasons why clinicians refused the research team access to patients. 'Gatekeeping' is not always inappropriate. Some gatekeeping by clinical staff is necessary to protect vulnerable patients from research that may be burdensome. More indepth research would have been required to tease out the extent to which gatekeeping in this study was appropriate or paternalistic.

\section{Relationship to other studies}

The first bottleneck to recruitment that we considered was the proportion of 'palliative care' patients who fulfilled the inclusion and exclusion criteria for the study. This is not a subject that has received much attention in previous reports. It is however apparent that if entry criteria are too proscriptive then recruitment will be difficult. Conversely if entry criteria are too lax then the sample population will be too heterogeneous for the purposes of the research. In the PiPS study the entry criteria were designed to capture the 'typical' cancer patient receiving palliative care. We had assumed that only a minority of patients under the care of palliative care services would still be on active cancer therapy. In fact we found that $26 \%$ (3259/12 412) of all patients referred to participating units were still on treatment.

One of the biggest problems facing palliative care researchers is gaining access to patients. In this study $66 \%$ of eligible patients were not seen by researchers. This study is one of the first to identify reasons for this. Some of the reasons for inaccessibility are not negotiable and are purely due to factors outside the control of the research team (eg, late referrals to the palliative care team, the rapid turnover of patients or the absence of a carer to provide assent for those patients who are deemed incompetent). However, researchers were unable to approach $16 \%(1101 / 7018)$ of eligible patients as frontline clinical staff denied them access. It has been reported that some staff consider it unethical to include patients who may be vulnerable ${ }^{11}$ in demanding research studies. Gatekeeping by staff has been identified as a significant issue affecting accrual to palliative care trials $^{3412}$ and a more common reason than patients' or 
families' refusing consent. ${ }^{13}$ While this is often borne out of a well-intentioned wish to protect patients and their families from further demands or stress, it denies patients their autonomy and may lead to sample bias. Healthcare professionals are reported to be reluctant to refer patients to studies which are complicated, involve randomisation or placebo controls, extra hospital visits or invasive procedures, ${ }^{3}$ or involve burdensome recordkeeping. ${ }^{12}$ Doctors sometimes have concerns regarding the impact of research on the doctor-patient relationship $^{14}$ or are averse to open discussions about uncertainty. ${ }^{15}$ Healthcare professionals are less likely to refer patients for pharmacological studies in which they may suffer side effects. ${ }^{3}$ Studies suggest that strategies to overcome gatekeeping include the design of studies that are relevant, quick and easy to do and not too demanding on patients. 41617

Studies exploring advanced cancer patients' views on participating in research have reported that they often refuse because they feel too unwell ${ }^{18}$ or are too symptomatic $^{19}$ to participate or simply that they are not interested or are unwilling to discuss research at this stage of their illness. ${ }^{8}$ Ling et $a l^{8}$ described patients feeling that they need more time to consider participation. Patients in the latter stages of illness may have poor cognitive function which makes consent more difficult. ${ }^{20}$ A concern that research participation will alter their relationship with their physician ${ }^{21}{ }^{22}$ has been described as a deterrent to some patients. The support of relatives and their clinician influences patients' decisions to consent or not. White et $a l^{22}$ reported that only just over half of interested patients would consent to a trial if their relatives were unsupportive. Patients are reported to be less willing to participate in studies involving randomisation for fear of drawing the dummy arm. ${ }^{21} 23$ Equally studies that involve relatively invasive procedures, high levels of record keeping ${ }^{12}$ or an increase in hospital admissions ${ }^{24}$ are also viewed less favourably by patients. Increased financial costs (eg, travel costs) and inconvenience were a deterrent to some but not all patients. ${ }^{22}$

The literature describes similar challenges in enrolling patients into palliative care trials in primary care $^{13}{ }^{25}$ hospice $^{12}$ and hospital settings. ${ }^{8} 24$ However, there is a paucity of literature describing whether the site or setting of the study influences recruitment into trials. Jordhoy et al ${ }^{17}$ recruited patients into the same study from hospital, outpatient and community settings. They were able to identify that recruitment in the community was poor but reasons for this were not given. Other studies have also reported that recruitment in community or primary care setting is especially difficult. ${ }^{13} 17$

\section{Unanswered questions and further research}

Not all patients who are eligible to participate in a research project will be willing or able to do so. This study has identified a number of reasons why patients were not accrued to a large multicentre observational palliative care study. Some sources of attrition are 'fixed' by the study design and can only be overcome by taking a more flexible approach to defining inclusion and exclusion criteria to clinical studies. Other barriers to recruitment (particularly the failure to access eligible patients) could be improved by better research infrastructure (thereby minimising losses due to researcher unavailability). One of the largest potentially remediable causes of under-recruitment is the phenomenon of gatekeeping. This study was able to demonstrate that gatekeeping is a significant cause of poor accrual and occurred least frequently among hospice inpatients. What this study was unable to discover is the extent to which gatekeeping was justified and the extent to which it was a paternalistic response by clinical staff to 'protect' their patients from research. More research is needed to probe the reasons underlying this phenomenon, the level of gatekeeping that should be considered acceptable and identifying methods to overcome it when appropriate.

Acknowledgements We would like to thank the following colleagues for their help with this study; Rehana Bakawala, Prof Mike Bennett, Teresa Beynon, Dr Cath Blinman, Dr Patricia Brayden, Helen Brunskill, Dr Kate Crossland, Dr Alison Cubbitt, Rachel Glascott, Anita Griggs, Anne Harbison, Deborah Hart, Dr Caroline Lucas, Dr Wendy Makin, Dr Oliver Minton, Dr Paul Perkins, Marek Plaskota, Katie Richies, Dr Susan Salt, Ileana Samanidis, Dr Margaret Saunders, Dr Jennifer Todd, Dr Catherine Waight, Dr Nicola Wilderspin, Dr Gail Wiley and Julie Young. We would also like to thank Prof

John Ellershaw for chairing the steering committee and Robert

Godsill for providing a service user perspective. Thanks to

Rosie Head for administrative support and data management. We would also like to thank the following hospices and palliative care units for their participation in the study; $\mathrm{St}$ John's Hospice (Lancaster), Gloucestershire Hospitals NHS Foundation Trust, The Pasque Hospice (Luton), Guy's and St Thomas' NHS Foundation Trust, Princess Alice Hospice (Esher), Bolton Hospice, St Catherine's Hospice (Crawley), St George's Hospital NHS Trust, Surrey and Sussex Healthcare NHS Trust, St Ann's Hospice Manchester, Christie Hospital Manchester, Nightingale Macmillan Unit (Derby), Trinity Hospice (London) and Trinity Hospice (Blackpool).

Contributions PCS, BG, VK, CT, LCK and SB contributed to the study conception and design. All authors contributed to the interpretation of data, the drafting or revising of the manuscript and final approval for publication. PCS acts as the guarantor for this manuscript.

Funding This study was funded by Cancer Research UK, grant number C11075/A6126. SB is funded by Macmillan Cancer Support and the NIHR CLAHRC (Collaborations for Leadership in Applied Health Research and Care) for Cambridgeshire and Peterborough.

Competing interests None.

Ethics approval Wandsworth COREC.

Provenance and peer review Not commissioned; externally peer reviewed.

Data sharing statement Data will be shared with the permission of the Principal Investigator. Only anonymised data will be released/shared. When the principal investigator is contacted with a request to share his data, the requestor will be asked to provide a brief research proposal on how they wish to use the data. It should include the objectives, what data are requested, timelines for use, intellectual property and publication rights, etc. This may form the basis of a Data Sharing Agreement. If 
the principal investigator has doubts over the scientific validity of the proposal or the requestor's ability to analyse/interpret data correctly, this will be discussed with the requestor. Any refusal to share data in such circumstances will be accompanied by a clear justification.

Open Access This is an Open Access article distributed in accordance with the Creative Commons Attribution Non Commercial (CC BY-NC 3.0) license, which permits others to distribute, remix, adapt, build upon this work non-commercially, and license their derivative works on different terms, provided the original work is properly cited and the use is non-commercial. See: http://creativecommons.org/licenses/by-nc/3.0/

Correction notice This article has been updated since it was published Online First. The sentence, "This was most likely to occur in hospice patients and least likely in the community (39\% hospice, $29 \%$ hospice, $13 \%$ community; $\mathrm{p}<0.000001$ )." has been corrected to read "This was most likely to occur in hospice patients and least likely in the community (39\% hospice, 29\% hospital, $13 \%$ community; $\mathrm{p}<0.000001)$."

\section{REFERENCES}

1 Grande GE, Todd CJ. Why are trials in palliative care so difficult? Palliat Med 2000;14:69-74.

2 Steinhauser KE, Clipp EC, Hays JC, et al. Identifying, recruiting, and retaining seriously-ill patients and their caregivers in longitudinal research. Palliat Med 2006;20:745-54.

3 White C, Gilshenan K, Hardy J. A survey of the views of palliative care healthcare professionals towards referring cancer patients to participate in randomized controlled trials in palliative care. Support Care Cancer 2008;16:1397-405.

4 White C, Hardy J. Gatekeeping from palliative care research trials. Progress Palliat Care 2008;16:167-71.

5 White C, Hardy J. What do palliative care patients and their relatives think about research in palliative care?-a systematic review. Support Care Cancer 2010;18:905-11.

6 Todd AMH, Laird BJA, Boyle D, et al. A systematic review examining the literature on attitudes of patients with advanced cancer toward research. J Pain Symptom Manage 2009;37:1078-85.

7 Rinck GC, van den Bos GA, Kleijnen J, et al. Methodologic issues in effectiveness research on palliative cancer care: a systematic review. J Clin Oncol 1997;15:1697-707.

8 Ling J, Rees E, Hardy J. What influences participation in clinical trials in palliative care in a cancer centre?. Eur J Cancer 2000;36:621-6.

9 Gwilliam B, Keeley V, Todd C, et al. Development of prognosis in palliative care study (PiPS) predictor models to improve prognostication in advanced cancer: prospective cohort study. BMJ 2011;343:d4920.

10 Preacher KJ. Calculation for the chi-square test: an interactive calculation tool for chi-square tests of goodness of fit and independence [Computer software]. 2001. http://quantpsy.org (accessed 17 Jul 2012).

11 Casarett DJ, Karlawish JH. Are special ethical guidelines needed for palliative care research? J Pain Symptom Manage 2000;20:130-9.

12 Ross C, Cornbleet MA. Attitudes of patients and staff to research in a specialist palliative care unit. Palliat Med 2003;17:491-7.

13 Ewing G, Rogers M, Barclay S, et al. Recruiting patients into a primary care based study of palliative care: why is it so difficult? Palliat Med 2004;18:452-9.

14 Fallowfield L, Ratcliffe D, Souhami R. Clinicians' attitudes to clinical trials of cancer therapy. Eur J Cancer 1997;33:2221-9.

15 Taylor K, Margolese R, Soskolne C. Physicians reasons for not entering eligible patients in a randomized clinical trial of surgery for breast cancer. N Engl J Med 1984;310:1363-67.

16 Cook AM, Finlay IG, Butler-Keating RJ. Recruiting into palliative care trials: lessons learnt from a feasibility study. Palliat Med 2002;16:163-5.

17 Jordhoy MS, Kaasa S, Fayers P, et al. Challenges in palliative care research; recruitment, attrition and compliance: experience from a randomized controlled trial. Palliat Med 1999;13:299-310.

18 Pautex S, Herrmann FR, Zulian GB. Is research really problematic in palliative care? A pilot study. J Pain Symptom Manage 2005;30:109-11.

19 Casarett D, Kassner CT, Kutner JS. Recruiting for research in hospice: feasibility of a research screening protocol. J Palliat Med 2004;7:854-60.

20 Dean RA, McClement SE. Palliative care research: methodological and ethical challenges. Int J Palliat Nurs 2002;8:376-80.

21 Mills EJ, Seely D, Rachlis B, et al. Barriers to participation in clinical trials of cancer: a meta-analysis and systematic review of patient-reported factors. Lancet Oncol 2006;7:141-8.

22 White CD, Hardy JR, Gilshenan KS, et al. Randomised controlled trials of palliative care-a survey of the views of advanced cancer patients and their relatives. Eur J Cancer 2008;44:1820-28.

23 Terry W, Olson LG, Ravenscroft P, et al. Hospice patients' views on research in palliative care. Intern Med J 2006;36:406-13.

24 Cox K, Avis M. Psychosocial aspects of participation in early anticancer drug trials. Report of a pilot study. Cancer Nurs 1996;19:177-86.

25 Murray S, Barclay S, Bennett MI, et al. Palliative care research in the community: it is time to progress this emerging field. Palliat Med 2008;22:609-11. 